\title{
Asignación óptima de recursos de comunicaciones para sistemas de gestión de energía
}

\section{(Optimal allocation of communications resources for energy management systems)}

\author{
Milton Ruiz, ${ }^{1}$ Esteban Inga ${ }^{2}$
}

\section{Resumen}

El presente artículo plantea la asignación óptima de recursos de los canales de radio disponibles en las redes celulares para la transmisión de datos desde los medidores inteligentes de energía eléctrica hacia los sistemas de gestión de información. El modelo planteado identifica y contabiliza a los abonados del sector eléctrico en un área geográfica; mediante el algoritmo de clusterización kmeans se ubican las radios bases celulares, las mismas que brindan comunicaciones a los dispositivos dentro de su radio de cobertura. Los canales de comunicación disponibles para la transmisión de datos son optimizados usando el algoritmo de Greedy, el mismo regula el tiempo en que los medidores inteligentes deben enviar su data a través de los canales de comunicación en desuso.

\section{Palabras clave}

Optimización; Greedy; k-means; comunicaciones celulares; redes eléctricas inteligentes.

\begin{abstract}
This article proposes the optimal resource allocation of radio channels available in cellular networks for the transmission of data from the smart meters to information management systems. The model designed identifies and counts the subscribers of the electricity sector in a geographical area; through the kmeans algorithm the cellular radio bases are located. The optimal location of radio bases provides communication to all devices within its coverage radius. The communication channels available for data transmission are optimized using the Greedy algorithm, which set the time when smart meters must send their data through the disused communication channels.
\end{abstract}

\section{Keywords}

Optimization; Greedy; k-means; cellular communications; smart grids

\section{Introducción}

La presente investigación es una extensión del artículo titulado "Optimal Communications for Smart Measurement of Electric Energy Reusing Cellular Networks" (Ruiz, Masache y Inga, 2018), presentado previamente en la Tercera Conferencia Internacional INCISCOS 2018 celebrada en la ciudad de Quito, Ecuador.

La red eléctrica inteligente comunica a numerosos sistemas con el propósito de brindar altos parámetros de seguridad, confiabilidad y disponibilidad empleando de manera eficiente los recursos necesarios para suministrar energía eléctrica sustentable respondiendo al crecimiento de la demanda.

La infraestructura de telecomunicaciones se nombran de acuerdo al área geográfica que suministra servicio, los mismos son conocidos como: 
- Red de área del hogar (HAN)

- Red de área de vecindario (NAN)

- Red de área extendida (WAN)

La red HAN presenta gran cantidad de dispositivos con comunicaciones y topologías heterogéneas (Maldonado, 2017) generando grandes volúmenes de información por lo que es necesario utilizar técnicas de reconstrucción como MapReduce (Inga Ortega, Inga, Correa y Hincapié, 2018). Una parte esencial de la red eléctrica inteligente es la infraestructura de medición avanzada, la misma requiere de equipos robustos que incorporan comunicaciones bidireccionales entre las empresas comercializadoras y los abonados, la gran cantidad de datos de los abonados del sector eléctrico son almacenados por las empresas de distribución por lo que es necesario una estructura de almacenamiento para Big Data (Moscoso Zea, 2012). La infraestructura de comunicación desplegada sobre la red de transporte eléctrico (Ruiz, Masache y Domínguez, 2018) permiten identificar y mitigar las fallas producidas de forma inmediata (Carrión, González y López, 2017) (Matos y Sanchez, 2016).

La Figura 1 muestra la aplicación de cada infraestructura red de comunicaciones, la red HAN se encuentra dentro de los hogares y comunica a dispositivos como autos eléctricos, lavadoras, interruptores, toma corrientes, etc. La comunicación entre los medidores inteligentes y los sistemas de gestión de datos son establecidos por las redes de vecindario NAN (por su sigla en inglés). El concentrador gestiona a los medidores inteligentes de forma automática, realiza el control, transfiere la información de diagnósticos y actualizaciones de firmware. A través de la red NAN se proporcionan servicios de transporte de datos de medición, monitoreo, control y detección de fallos(Gungor et al., 2013). Finalmente, la red WAN transporta la información desde los medidores hacia los centros de gestión de información en cada empresa eléctrica de distribución (Ekanayake y Liyanage, 2012).

Figura 1. Infraestructura de Comunicación para medición inteligente

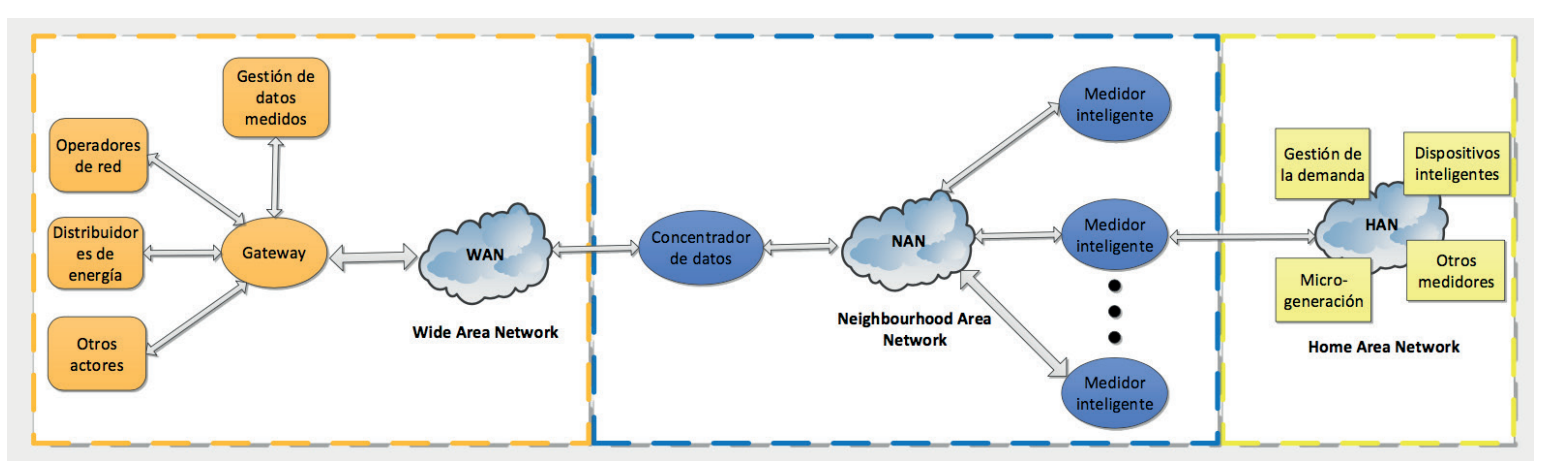

\section{Parámetros de Diseño de la Infraestructura de Red}

La integración de infraestructura de telecomunicaciones al sistema eléctrico permite el flujo de información entre todos los actores del sector eléctrico. AMI provee medición en tiempo real, detección de fallas y la participación del usuario. La información colectada por los medidores es enviada a los sistemas de gestión de datos de medidores MDMS (por su sigla en inglés). Los sistemas de gestión de datos brindan almacenamiento y procesamiento de información(Arciniegas, Imbajoa y Revelo, 2017). 
Para el diseño de una red inalámbrica es necesario tomar en cuenta requerimientos como cobertura, capacidad, latencia y seguridad con la finalidad de obtener una red de comunicación inalámbrica eficiente. Estándares inalámbricos como IEEE 802.11, IEEE 802.15.4, 3G/LTE y IEEE 802.16 son utilizados para dar servicio a medición inteligente.

La Tabla 1 muestra las características de cada tecnología celular empleada para la transmisión de información de los medidores inteligentes hacia los sistemas de gestión de información.

Tabla 1. Tecnologías celulares

\begin{tabular}{|l|l|l|l|l|}
\hline Tecnología & \multicolumn{1}{|c|}{ Espectro } & \multicolumn{1}{|c|}{ Tasa } & \multicolumn{1}{c|}{ Cobertura } & \multicolumn{1}{c|}{ Aplicaciones } \\
\hline GSM & $900-1800 \mathrm{MHz}$ & Hasta $14.4 \mathrm{kbps}$ & $1-10 \mathrm{~km}$ & AMI, HAN \\
\hline GPRS & $900-1800 \mathrm{MHz}$ & Hasta $170 \mathrm{kbps}$ & $1-10 \mathrm{~km}$ & AMI, HAN \\
\hline $3 G$ & $1.92-1.98 \mathrm{GHz} 2.11-2.17 \mathrm{GHz}$ & $384 \mathrm{kbps} 2 \mathrm{Mbps}$ & $1-10 \mathrm{~km}$ & AMI, HAN \\
\hline WiMAX & $2.5 \mathrm{GHz}, 3.5 \mathrm{GHz}, 5.8 \mathrm{GHz}$. & Hasta $75 \mathrm{Mbps}$ & $\begin{array}{l}10-50 \mathrm{~km}(\mathrm{LOS}) \\
1-5 \mathrm{~km} \text { (NLOS) }\end{array}$ & AMI, HAN \\
\hline
\end{tabular}

Las redes celulares cubren grandes extensiones geográficas con gran eficiencia en el uso del espectro electromagnético, reutilizando los canales de radio y reasignándolos a varias celdas ubicadas en diferentes áreas de cobertura conocidas como celdas co-canal. La interferencia entre celdas es amortiguada por la separación co-canal en las celdas. Cada celda está controlada por un transductor llamado estación base por sus siglas BS (Zhang, Liu y Zeng, 2017).

La mayor infraestructura celular desplegada es conocida como 3G, proporciona comunicaciones inalámbricas de alta velocidad para aplicaciones multimedia, datos, video y voz. Entre las principales características se estableció 144 kbps de velocidad de datos para usuarios que se movilizan a altas velocidades, $384 \mathrm{kbps}$ de velocidad de datos para usuarios a baja movilidad y 2 Mbps para uso de oficina(Ruiz, 2016).

La Figura 2 muestra la topología de red celular sobre la cual se plantearán los diseños de optimización de asignación de recursos.

Figura 2. Infraestructura Celular

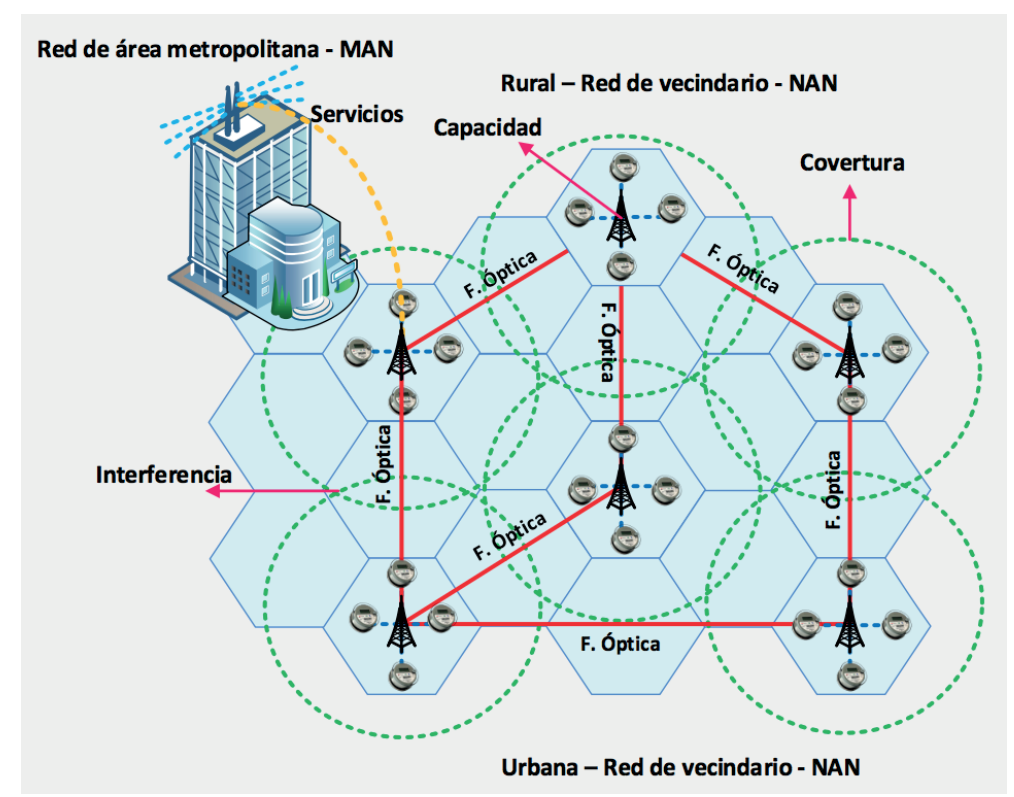


Actualmente se están desplegando comunicaciones móviles de cuarta generación y quinta generación conocidas como 4G o LTE-Advanced y 5G. Las redes celulares de cuarta generación ofrecen acceso móvil a internet de banda ancha para teléfonos inteligentes y otros dispositivos móviles. La tecnología $4 \mathrm{G}$ se refiere a las IMT-Avanzadas (Telecomunicaciones Móviles Internacionales-Avanzadas), definida por el ITU-R. Un sistema celular IMT-Advanced debe tener velocidades de datos pico de hasta aproximadamente $100 \mathrm{Mbps}$ para alta movilidad y aproximadamente 1Gbps para baja movilidad, traspasos suaves a través de redes heterogéneas y la capacidad para ofrecer alta calidad de servicio para el soporte multimedia de última generación (Ekpenyong, Isabona y Isong, 2016).

La cantidad de recursos dedicados para establecer las conexiones entre los medidores, concentradores y radio bases se ve afectada debido a la cantidad de dispositivos a los que se debe dar servicio evitando colisiones y a los intentos de conexión realizados por las peticiones de retransmisión. Estos problemas afectan al tiempo de espera para acceder a los canales de control y de datos.

La reutilización de frecuencias permite aumentar la capacidad del sistema celular utilizando de manera eficiente el espectro radioeléctrico.

La Ecuación 1 permite calcular el número de canales dúplex disponibles. Se debe tener en cuenta que al dividir los canales disponibles se forman grupos de canales únicos disjuntos y cada grupo posee el mismo número de canales.

$$
\boldsymbol{S}=\boldsymbol{k} \times \boldsymbol{N}
$$

Donde: $\mathrm{S}$ = total de canales dúplex, $\mathrm{k}=$ número de canales por grupo asignados a cada celda $(\mathrm{k}<\mathrm{S}), \mathrm{N}=$ número de celdas entre las cuales se dividen los $\mathrm{S}$ canales.

La Ecuación 2 permite calcular la capacidad del sistema celular tomando en cuenta que la capacidad es directamente proporcional al número de veces que un clúster se replica en un área de servicio fija.

$$
\boldsymbol{C}=\boldsymbol{M} \times \boldsymbol{S}
$$

Donde $C$ = capacidad del sistema, $M$ = número de veces que se replica un clúster .

El tamaño del clúster es denominado con la letra N y sus valores típicos son cuatro, siete y doce. El valor del clúster es una función de cuanta interferencia una estación base o un móvil puede tolerar manteniendo calidad en las comunicaciones.

Para calcular el tamaño del clúster y el arreglo de las celdas se considera la geometría hexagonal, es decir cada celda tiene exactamente seis vecinos equidistantes y las líneas que unen los centros de cada celda están separadas sesenta grados.

La Ecuación 3 permite encontrar el co-canal vecino más cercano de una celda en particular.

$$
N=i^{2}+i j+i^{2}
$$

Donde $\mathrm{i}=$ número entero positivo, $\mathrm{j}$ = número entero positivo

La selección de redes privadas con infraestructura dedicada para AMI implica grandes costos y son difíciles de financiar, implementar, mantener y ejecutar. Mientras que la selección de redes públicas requiere menor inversión de capital en comparación con una red dedicada, dando como resultado un menor impacto económico. Se debe tomar en cuenta que, en la red privada, el proveedor de servicios es responsable de garantizar el rendimiento, la seguridad y 
la actualización de la infraestructura. Por otra parte, la participación de múltiples proveedores públicos y privados puede mejorar la tolerancia a fallos o extender la cobertura.

Las transmisiones inalámbricas se ven afectadas ya que todos los medidores utilizan los mismos canales de radio y, por lo tanto, la misma frecuencia de transmisión y recepción. Se debe considerar que el uso de bandas no licenciadas aumenta la interferencia y el ruido por las comunicaciones externas al sistema de medición avanzada.

La Tabla 2 presenta los requerimientos mínimos que deben cumplir las tecnologías de comunicación para la medición inteligente, tomando en cuenta que deben ser capaces de gestionar la información de cientos de miles de medidores inteligentes.

Tabla 2. Requisitos para AMI

\begin{tabular}{|l|l|}
\hline \multicolumn{1}{|c|}{ Requerimiento } & \multicolumn{1}{c|}{ Rango } \\
\hline Ancho de banda & $10-100 \mathrm{kbps} /$ nodo \\
\hline Latencia & $2-15$ segundos \\
\hline Confiabilidad & $99-99.99 \%$ \\
\hline Seguridad & Alta \\
\hline Respaldo de Energía & No es necesario \\
\hline
\end{tabular}

La generación de datos depende del tiempo de monitoreo configurado en el sistema, por lo tanto, pueden existir muchos sensores generando información y transmitiendo a su nodo vecino hasta llegar al concentrador de la red. Del mismo modo los sensores que se encuentran de puente de comunicaciones entre otros sensores pueden tener información almacenada por enviar y debe tener la suficiente memoria para tomar los datos y retransmitirlos, así como para transmitir sus datos generados.

Los diferentes sub-canales experimentan diferentes ganancias, incluso para el mismo usuario. Cada sub-canal es lo suficientemente estrecho como para sufrir un desvanecimiento. También asumimos que la ganancia del canal es constante en cada ranura de tiempo, pero varía de una ranura a otra. Bajo las suposiciones anteriores la Ecuación 4 permite calcular la señal de ruido en función de la ganancia del canal calculado por la Ecuación 5.

$$
\begin{gathered}
\mathrm{SNR}=\frac{S_{k, n}(t)^{*} H_{k, n}(t)}{N^{*} \frac{B}{N}} \\
H_{k, n}(t)=\sum_{n=0}^{N-1} H_{k, n}(t)^{2} / N
\end{gathered}
$$

De donde; SNR, es la señal ruido; H es la ganancia del canal; S es la potencia transmitida;

Un enfoque amplio para la simulación de canales es construir una señal a partir de fuentes de ruido gaussiana, si un canal está compuesto por un conjunto de componentes de señales discretas que se originan por la reflexión o dispersión de estructuras más pequeñas, por ejemplo, casas, pequeñas colinas, etc., se denomina un canal multitrayecto discreto. La respuesta equivalente de un canal multitrayecto se obtiene con la Ecuación 6.

$$
C(t)=\sum_{k=1}^{k(t)} a_{k}(\tau(t), t)^{*} \delta(\tau-t)
$$


Donde $\tau$ es el tiempo.

Para muchos canales, se puede suponer como una aproximación razonable de que el número de componentes discretos es constante y los valores de retardo varían muy lentamente y también pueden ser constantes. El modelo entonces se simplifica en la Ecuación 7.

$$
C(t)=\sum_{k=1}^{k(t)} a_{k}(t)^{*} \delta(\tau-t)
$$

Donde $a_{k}(t)$ y $\delta\left(\tau-\tau_{k}\right)$ son la atenuación y el retardo.

Al utilizar comunicaciones celulares y con la finalidad de brindar calidad de servicio se determina la pérdida del servicio mediante Erlang B (Ecuación 8), debido a que los sistemas de telefonía en redes fijas y móviles que no proporcionan almacenamiento en búfer de tráfico.

$$
P_{b}=B(A, m)=\frac{\frac{A^{m}}{m !}}{\sum_{i=0}^{m} \frac{A^{i}}{i !}}
$$

Para los cálculos de tráfico se ha tomado en cuenta la tecnología celular GSM desplegada por una operadora celular en el país. La Tabla 3 describe las principales características de la tecnología celular GSM.

Tabla 3. Características de GSM

\begin{tabular}{|l|l|}
\hline \multicolumn{1}{|c|}{ Descripción } & \\
\hline Método de acceso & TDM/TDMA \\
\hline Canales full-duplex & 125 \\
\hline Modulación & GMSK \\
\hline Usuarios por canal & 8 \\
\hline Tasa de bits por ranura & $22.8 \mathrm{kbps}$ \\
\hline Tasa de bits total & $270.8 \mathrm{kbps}$ \\
\hline
\end{tabular}

\section{Formulación del problema}

Para calcular el tráfico generado por la medición inteligente es necesario tomar en cuenta el número de abonados registrados al servicio eléctrico a nivel nacional. Los abonados al servicio eléctrico a nivel nacional bordean los 3'359.400. Se debe tomar en cuenta que el tiempo máximo entre mediciones es de 15 minutos por cada medidor y los datos generados pueden ser transmitidos en mensajes cortos de texto o llamada celular.

Al utilizar SMS en el envío de información entre el MDMS y los medidores es necesario conocer que todos los mensajes cortos de texto ingresan a la infraestructura celular y son procesados por el centro de servicios de mensajes cortos por sus siglas en ingles SMSC, el mismo que se encarga de almacenar y transmitir los mensajes hasta los destinatarios. Los SMS adicionan información a cada mensaje de texto con parámetros como la fecha de envío, la validez del mensaje, el SMSC que origino el mensaje y el número de identificación del remitente y destinatario. Al ser mensajes cortos de texto no es necesario la asignación de canales de radio 
por lo que se los puede enviar en los canales de señalización incluso se pueden enviar y recibir mientras existe una llamada en curso.

Para calcular el número de SMS que se enviarán en un año por medición de energía eléctrica se ha establecido mensajes cada 15 minutos durante las 24 horas los 365 días del año, la cantidad de información anual generada es de 39'244.800 bits con un total de 35.040 lecturas. Si restamos los caracteres delimitadores de cada palabra tenemos que la información útil es de $27^{\prime} 471.360$ bits.

El tiempo de uso del canal se calcula dividiendo la cantidad de bits sobre la tasa de transmisión de GSM dando como resultado 0,0491 segundos.

La cantidad de tráfico generada por cada medidor se calcula multiplicando el número de mensajes enviados cada hora por el tiempo de uso del canal, cada medidor genera 0,0000546 Erlangs/hora. El tráfico total generado durante una hora por los medidores inteligentes es la multiplicación del número de medidores por el tráfico de cada medidor, en total se genera 183 Erlangs/hora.

Provincias como Pichincha requieren 32,7 canales del servicio celular para realizar medición inteligente, tomando en cuenta que en la provincia de Pichincha existen 799 radio bases GSM instaladas por una de las tres operadoras. La capacidad brindada por la operadora celular se calcula tomando en cuanta cuenta 125 canales full dúplex y tamaño de clúster de 7 . Se estima que existen 114.143 canales de radio que prestan servicio de voz y datos. Con la finalidad de brindar calidad de servicio a los datos adquiridos por los medidores inteligentes se calcula la cantidad de canales necesarios para brindar grados de servicio de 99 \% y 99.9. Al utilizar Erlang B se estima que son necesarios 45 y 51 canales del servicio celular respectivamente.

El algoritmo propuesto permite la creación de redes inalámbricas optimizando el tráfico de información, para lo cual se ha planteado un escenario con 500 medidores dentro de un área de cobertura de $1 \mathrm{~km}^{2}$.

El Algoritmo 1 ubica a las radiobases celulares que brindan conexión inalámbrica entre los medidores y la estación más cercana para el envío de la información. El algoritmo K-means realiza un agrupamiento de los medidores a la radio base más cercana, la ubicación va a hacer designada como una conexión fija.

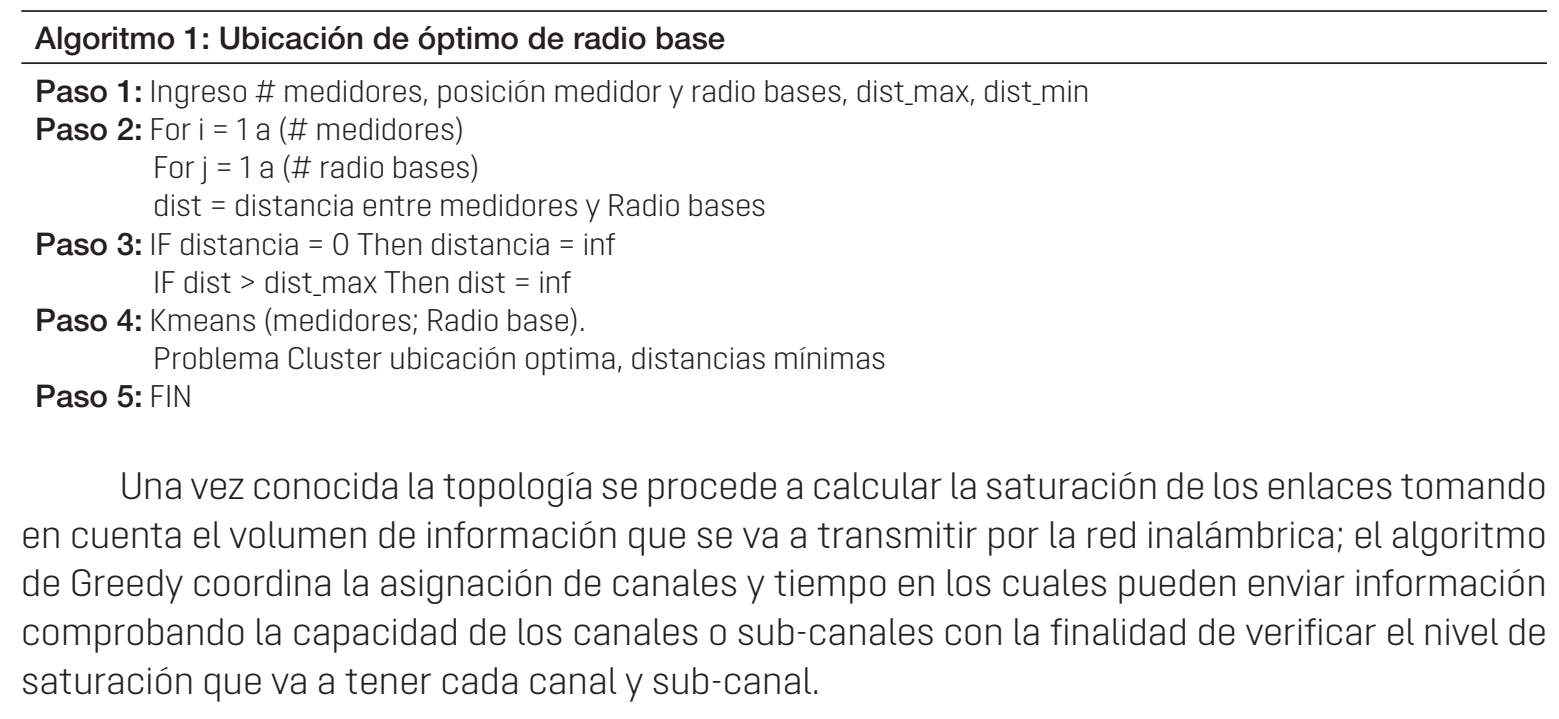


El Algoritmo 2 planteado divide el problema de la asignación en dos pasos. En el primer paso, el número de sub-canales que se le asignará a los medidores se determina en función de los requisitos de cantidad de datos enviados por los usuarios y de la señal de ruido promedio. Se muestra el algoritmo para encontrar la distribución de sub-canales que minimiza el uso total del canal. En la segunda etapa del algoritmo, encuentra la mejor asignación de sub-canales a los medidores según la tasa estimada de transmisión. El objetivo es encontrar la asignación de sub-canales que permita a cada dispositivo satisfacer el envío de información.

En la Tabla 4 se detallan los parámetros requeridos por el algoritmo Greedy para la optimización de los recursos de comunicación. Los parámetros mostrados son establecidos en base a la red celular 3G, la misma que presenta el mayor despliegue a nivel nacional por las operadoras del país.

Tabla 4. Parámetros de ingreso del algoritmo

\begin{tabular}{|l|l|}
\hline \multicolumn{2}{|c|}{ Información del algoritmo Greedy } \\
\hline Velocidad de transmisión & $22.8 \mathrm{kbps}$ \\
\hline Número de Canales & 3 \\
\hline Frecuencia simplex & $201.6 \mathrm{Khz}$ \\
\hline Numero de equipo & 500 \\
\hline Sub-canal & $8 \mathrm{c} / \mathrm{u}$ \\
\hline Tiempo de muestreo & $15 \mathrm{~min}$ \\
\hline Tecnología & GSM \\
\hline
\end{tabular}

Asignación de usuarios: se asigna la cantidad de sub-canales según los requisitos de velocidad y la ganancia de los usuarios.

Asignación de sub-canales: utiliza el resultado de la etapa de asignación de recursos y la información del canal para asignar los sub-canales, dependiendo de la transmisión de bits enviados, asumiendo que cada medidor envía un máximo de datos.

Al resolver estos problemas secundarios por separado, se encuentra una solución adecuada, pero no necesariamente óptima, que garantiza un nivel de servicio adecuado para cada medidor.

Todo lo expuesto en la metodología es usado como parámetros de ingreso para este algoritmo, es decir que los parámetros de ingreso van a hacer el cálculo de la interferencia, ruido producido en el canal, capacidad del canal.

A continuación, se describe el Algoritmo planteado.

\footnotetext{
Algoritmo 2: Asignación de recursos

Paso 1: Ingreso canal, sub-canal, Tiempo uso canal, velocidad de envío, frecuencia de muestreo, número de medidores

Paso 2: Calculo de Interferencia, ruido por el teorema de Shannon.

Paso 3: Greedy asignación de usuarios a nos sub-canales

Primera asignación-Problema Primario

Paso 4: Greedy comprobación de capacidad de los subcanales -Segunda asignación- Problema secundario

Paso 5: FIN
} 


\section{Resultados y discusión}

El comportamiento del algoritmo propuesto ha sido analizado en base a la creación de poblaciones aleatorias georreferenciadas con datos obtenidos desde Open Street Map. Los datos son descargados en formato.OSM y contiene las ubicaciones de domicilios, calles principales y secundarias así como sus respectivas longitudes y latitudes. Cada domicilio representa un medidor inteligente y las radio bases celulares son ubicadas utilizando el algoritmo de optimización.

La Figura 3 muestra cómo se realiza un enrutamiento óptimo, cuya principal variable va hacer la distancia euclidiana entre el equipo de medición y la estación base. De acuerdo a lo expuesto y tras varias simulaciones se determina que el número aproximado de equipos conectados a la estación base van a hacer entre 400 y 500 equipos promedio.

Figura 3. Despliegue óptimo de las radio bases celulares

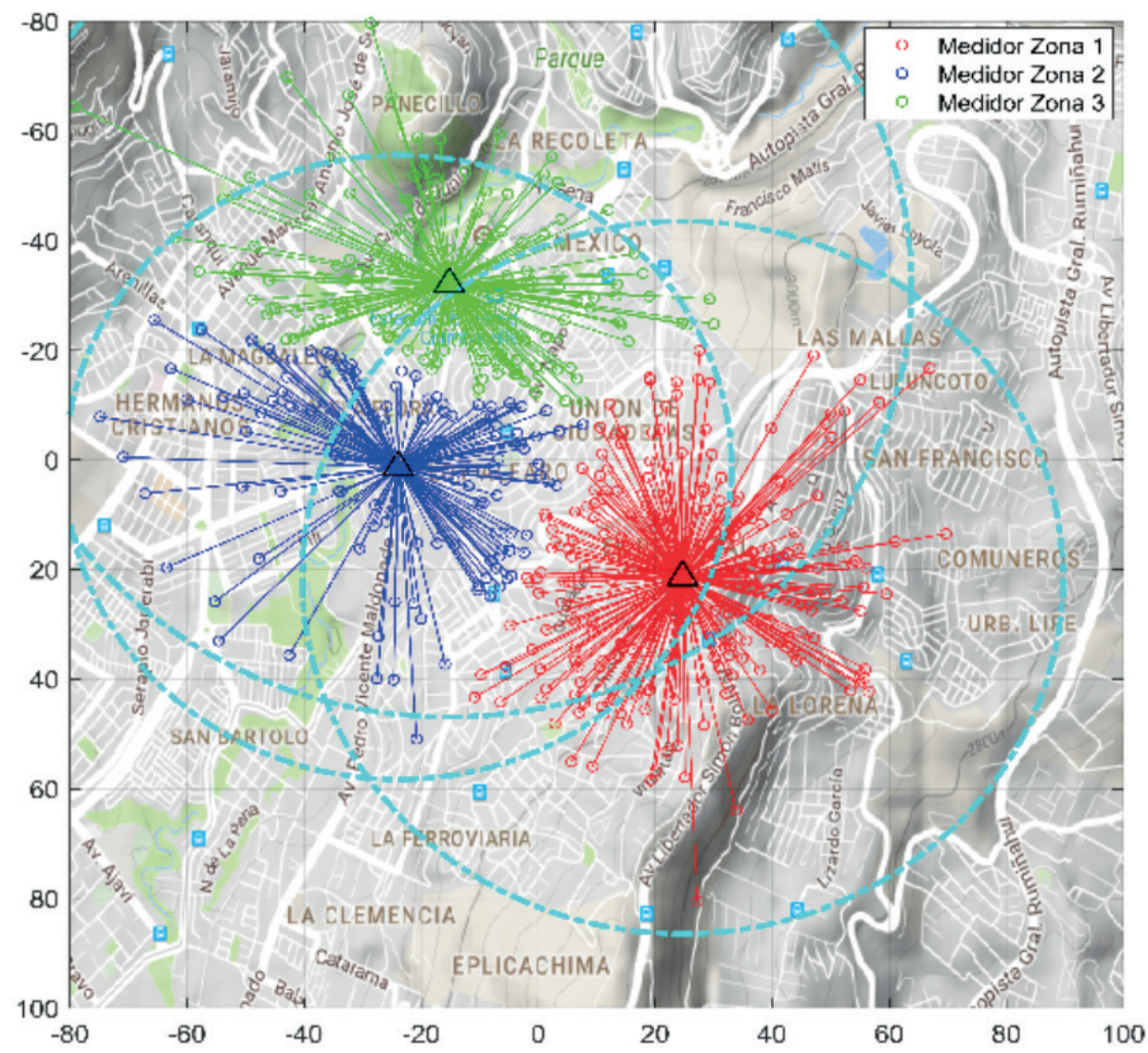

La Figura 4 presenta el uso de los canales y sub-canales sin optimización. Se observa que el número de canales asignados brinda cobertura a 500 usuarios y los datos son enviados desde cada medidor con un comportamiento aleatorio, esto implica que existen varios canales congestionados y otros canales presentan poco uso. La simulación envía datos cada 15 minutos, se utilizan 4 canales y 32 sub-canales que representa la multiplexación por división de tiempo. 
Figura 4. Asignación de los medidores a los canales

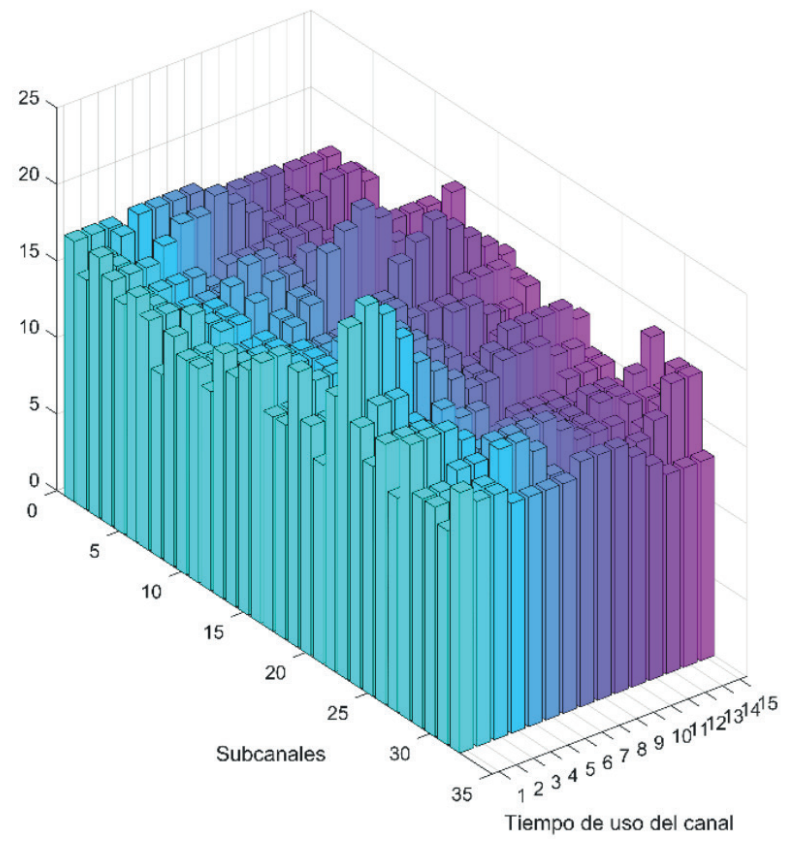

La Figura 5 presenta el resultado de la distribución uniforme de los canales y sub-canales realizado por el algoritmo propuesto. La red celular brinda servicio a 500 medidores inteligentes. Se observa que el algoritmo propuesto designa el momento en el que cada medidor debe realizar la transmisión de sus datos, dependiendo de la capacidad de cada uno de los canales, al mismo tiempo, se verifica la congestión y se utilizan los canales disponibles.

Figura 5. Capacidad de los canales

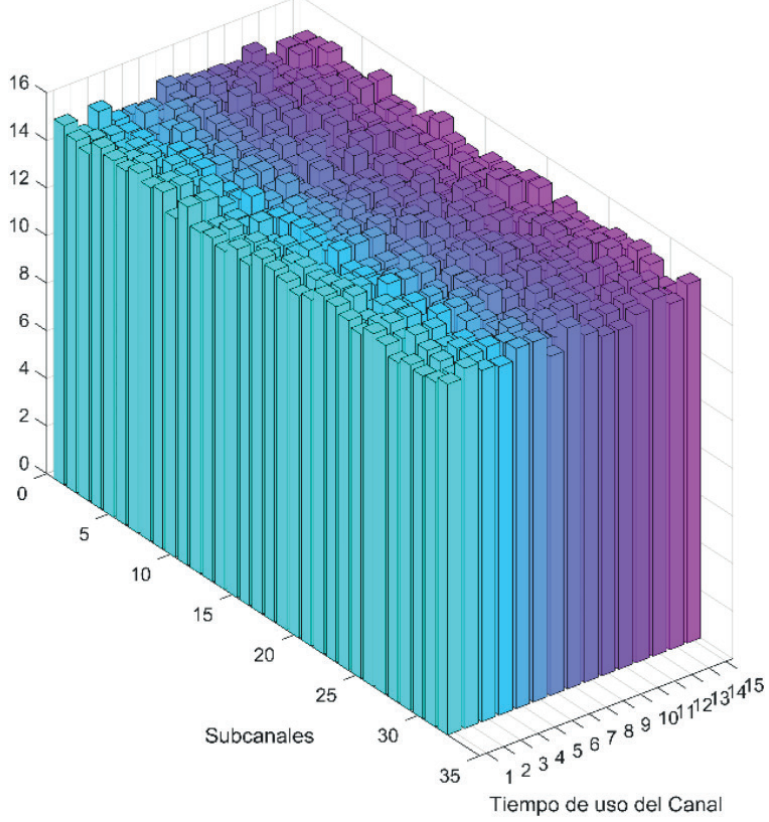




\section{Conclusiones y recomendaciones}

Los algoritmos planteados demuestran la posibilidad de optimizar el uso de los canales de radio comunicación en redes celulares mediante una estrategia de ubicación de radio bases en función de capacidad y cobertura.

La asignación de tiempos para envío de información desde los medidores inteligentes optimiza el uso de los canales de radio subutilizados.

La investigación demuestra que es posible utilizar las tecnologías celulares relegadas para la transmisión de datos desde los medidores inteligentes a los centros de gestión de información demostrando que no existe impacto en la capacidad del sistema celular debido a que los datos pueden ser transmitidos mediante mensajes cortos de texto SMS.

Futuros trabajos, plantearán un análisis para optimizar los datos que se transmiten desde los medidores a los centros de gestión utilizando técnicas de sensado comprimido para minimizar el volumen de información transmitida y almacenada.

\section{Referencias}

Arciniegas, A. F., Imbajoa, D. E., y Revelo, J. (2017). Diseño e implementación de un Sistema de Medición Inteligente para AMI de la microrred de la Universidad de Nariño (Design and implementation of a Smart Measurement System for AMI in the microgrid of the University of Nariño). Enfoque UTE, 1, 300-314. Retrieved from http://ingenieria.ute.edu.ec/enfoqueute/

Carrión, D., González, J. W., y López, G. J. (2017). Optimal Fault Location in Transmission Lines Using Hybrid Method. 2017 IEEE PES Innovative Smart Grid Technologies Conference, 6. https://doi. org/10.1109/ISGT-LA.2017.8126757

Ekanayake, J., y Liyanage, K. (2012). Smart Grid Technology and Applications. Nueva Delhi: Wiley.

Ekpenyong, M., Isabona, J., y Isong, E. (2016). Handoffs decision optimization of mobile celular networks. Proceedings-2015 International Conference on Computational Science and Computational Intelligence, CSCI 2015, (ii), 697-702. https://doi.org/10.1109/CSCI.2015.155

Gungor, V. C., Sahin, D., Kocak, T., Ergut, S., Buccella, C., Cecati, C., y Hancke, G. P. (2013). A Survey on smart grid potential applications and communication requirements. IEEE Transactions on Industrial Informatics, 9 (1), 28-42. https://doi.org/10.1109/TII.2012.2218253

Inga Ortega, E., Inga, J., Correa, E., y Hincapié, R. (2018). Reconstrucción del patrón de consumo eléctrico a partir de Big Data mediante técnica de MapReduce. Enfoque UTE, 9(1), 177-187. https://doi. org/10.29019/enfoqueute.v9n1.220

Maldonado, M. G. R. (2017). Wireless Sensor Network for Smart Home Services Using Optimal Communications. 2017 International Conference on Information Systems and Computer Science (INCISCOS), 27-32. https://doi.org/10.1109/INCISCOS.2017.21

Matos, L. O., y Sanchez, J. W. G. (2016). Reconfiguration strategy for Fault Tolerance of power Distribution Systems using Petri net. 2016 IEEE Ecuador Technical Chapters Meeting, ETCM 2016, 1-6. https://doi.org/10.1109/ETCM.2016.7750820

Moscoso Zea, O. (2012). Megastore: structured storage for Big Data. Enfoque UTE, 3(2), 01. https://doi. org/10.29019/enfoqueute.v3n2.1

Ruiz, M. (2016). Diseño de un sistema híbrido inalámbrico-fibra para transmisión de datos de medidores inteligentes de energía en redes Smart Grid. Pontificia Universidad Católica del Ecuador. Retrieved from http://repositorio.puce.edu.ec/handle/22000/11293

Ruiz, M., Masache, P., y Domínguez, J. (2018). High Availability Network for Critical Communications on Smart Grids, (Ssn), 1-5. 
Ruiz, M., Masache, P. y Inga, E. (2018). Optimal Communications for Smart Measurement of Electric Energy Reusing Cellular Networks. 2018 International Conference on Information Systems and Computer Science (INC/SCOS), 198-204. https://doi.org/10.1109/INCISCOS.2018.00036

Zhang, Z., Liu, F., y Zeng, Z. (2017). The cell zooming algorithm for energy efficiency optimization in heterogeneous cellular network. 2017 9th International Conference on Wireless Communications and Signal Processing, WCSP 2017 - Proceedings, 2017-January, 1-5. https://doi.org/10.1109/ WCSP.2017.8171171 